PICU transmissions (patients/observational period $2-3-4-$ $2-1)$.

Conclusion Aplying the international guidelines in to everydaylife practice did not result in increased morbidity. Besides there was a positive effect on cost effectivity and patient comfort.

\section{P554 A REVIEW OF THE USE OF THE PNEUMOCOCCAL URINARY ANTIGEN PROTEIN AS A DIAGNOSTIC BIOMARKER IN THE PAEDIATRIC POPULATION AT UNIVERSITY HOSPITAL LIMERICK OVER THE LAST TWO YEARS}

${ }^{1}$ Niall Dunworth* ${ }^{1}$ Katie Flinn, ${ }^{1}$ Niamh Daly, ${ }^{2}$ Nuala O'Connell, ${ }^{1}$ Barry Linnane. ${ }^{1}$ Department of Paediatrics, University Hospital Limerick, Limerick, Ireland; ' ${ }^{2}$ Department of Microbiology, University Hospital Limerick, Limerick, Ireland

10.1136/archdischild-2019-epa.888

Background The 'pneumococcal urinary antigen protein' assay is a commonly used, and widely accepted, aide in diagnosing pneumonia amongst adult patients. There is little evidence for its use in the paediatric population and it is not validated for use in patients 6 years of age and younger. Nevertheless, there has been an emerging trend in its use at our hospital in the paediatric setting. Interestingly, there seems to have been significant positive correlation between a positive result and clinical presentations consistent with Community Acquired Pneumonia (CAP).

Aim Our aim is to review the frequency of pneumococcal urinary antigen testing in the paediatric population over the last two years at UHL. We are assessing the relationship between a positive result and other markers of inflammation (WBC, Neutrophilia, Elevated CRP, Elevated Platelet count, Decreased serum albumin), the presence of consolidation on Chest $\mathrm{X}$ Ray, vital signs at presentation in ED, first line antibiotic therapy prescribed, need for second line antibiotics and the presence of associated complications. Specifically, we endeavour to determine if a positive result in children and adolescent patients relates to a clinical outcome consistent with CAP.

Methods To search for all patients 16 years of age and under who had a positive result using the iLab software. iLab will also be used to assess other markers of inflammation, in the same patient cohort, at time of admission. Corresponding chest radiograph images and reports will be accessed via the NIMIS radiology software. Vital signs at time of presentation will be assessed using the 'Therefore' software. Information regarding antibiotic treatment and any complications can be accessed using the hospital's E-discharge summary system

Results There were X paediatric patients who had their pneumococcal urinary antigen protein tested in UHL from January 2017 to December 2018 inclusive, X of which tested positive. Of the positively resulted cohort, $\mathrm{X}$ had evidence of consolidation on CXR, X had corresponding markers of inflammation at $\mathrm{ED} . \mathrm{X} \%$ of patients required antibiotic therapy and a further $\mathrm{X} \%$ required second line antibiotic therapy. $\mathrm{X}$ patients had clinical features of acute infection at presentation and $\mathrm{X}$ amount of patients had associated complications.

Conclusion The results of this review indicate a correlation between a positive result and clinical presentation of CAP. This may well support the use of the pneumococcal urinary antigen protein assay as part of the work up for paediatric patients presenting with symptoms concerning for CAP.
P555 A RARE CASE OF CONGENITAL LOBAR OVERINFLATION

Jennifer Finnegan*, John David Corcoran, Ailbhe Tarrant. Rotunda Hospital, Dublin, Ireland

\subsection{6/archdischild-2019-epa.889}

Background AF was born by normal vaginal delivery at $38+4$ weeks gestation. A normal antenatal scan was performed at 22 weeks. Septic workup was performed and antibiotics commenced due to PROM and GBS carriage in the mother.

Clinical course and imaging

She was admitted to NICU with tachypnoea and increased work of breathing at three hours of age. Ambient incubator oxygen up to $33 \%$ was commenced to maintain $\mathrm{O}_{2}$ saturations above 94\%. The initial working diagnosis was of TTN. Initial chest $\mathrm{x}$-ray showed a left lower lobe opacity and left pleural effusion. Oxygen was weaned and discontinued. Repeat imaging on day 3 showed left retrocardiac density and left lower lobe segmental atelectasis. The left upper lobe and lingula demonstrated hyperinflation. A decision was made to treat with IV antibiotics for 5 days for a suspected congenital pneumonia.

Imaging was repeated on day 4 due to deterioration in clinical condition with increasing tachypnoea and a further requirement for oxygen. Hi-flow oxygen was commenced, with $\mathrm{FiO}_{2}$ of $35 \%$. There was a further increase in hyperlucency of left upper lobe with contralateral midline shift and left lower lobe collapse. A lateral x-ray confirmed that hyperlucency was due to overexpansion and suggestive of congenital lobar overinflation (CLO) rather than pneumothorax.

Management High-frequency oxygen was discontinued and she remained on ambient $\mathrm{O}_{2}$ and was nursed right side up. She remained in a stable condition until transfer to a tertiary hospital where CT thorax confirmed the diagnosis. She awaits pulmonary lobectomy.

Congenital lobar overinflation CLO is a rare disorder characterised by hyperinflation of one or more pulmonary lobes. It has a prevalence of 1 in 20,000 to 1 in 30,000 . It most commonly affects the left upper lobe. A narrow bronchus causes collapse and air trapping during expiration. Cases can be diagnosed antenatally during foetal ultrasound. Although 50\% of cases appear in the newborn period, symptoms of tachypnoea can develop into childhood. Chest $\mathrm{x}$-ray and CT are diagnostic in full-term, non-ventilated babies. X-ray typically shows increased density in the affected lobe initially, transitioning to hyperlucency as the affected lobe overinflates. The CT confirms the lobar overinflation, absence of bullae etc, and evaluates for the possibility of an aberrant mediastinal vessel as a potential cause. Lobectomy of affected lobe is a widely accepted treatment.

Conclusion CLO is typically associated with progressive respiratory distress and mediastinal shift.

Although rare, CLO should remain a differential for respiratory distress in a term neonate.

\section{P556 IMPORTANCE OF LUNG ULTRASOUND IN PEDIATRICS}

Shendvera Hasani*, Drita Qosaj. University Clinical Center, Prishtina, Albania

10.1136/archdischild-2019-epa.890

Acute respiratory conditions are frequent conditions in children which cause sudden respiratory function deterioration 
also known as dyspnea. The exact determining of etiology is still a main diagnostic challenge.

Standard methods include: taking a history, clinical examination, auscultation and radiological procedures. But for many reasons, such as exposure to ionizing radiation and the inability to apply at bedside the chest radiography is not any more 'Gold standard' to acute respiratory insufficiency.

Lung ultrasound (LUS) has been shown to have great sensitivity and specificity in the differential diagnosis of the most common respiratory conditions. This method can be applied bedside, it can be used even outside of hospitals, follow - up is easy and does not expose the patient to harmful ionizing radiation.

According to a diagnostic algorithm called 'BLUE protocol' established by Daniel Lichtenstein lung ultrasound has been proven to be accurate in finding the true cause of respiratory insufficiency in a large number of cases.

Major acute respiratory disorders are pulmonary edema, pulmonary inflammation, acute respiratory distress syndrome (ARDS), pulmonary embolism, asthma exacerbation and chronic obstructive pulmonary disease (COPD), pleural effusion and pneumothorax.

Aim To demonstrate the role of lung ultrasound (LUS) as the first line of pulmonary condition diagnosis, to define the role of LUS during folow-up visits in order to prevent complications, to increase awareness of LUS importance in relation to the most frequent pulmonary disease in pediatric patients.

Materials-methods We have described the LUS examination technique as normal LUS images and LUS findings in the most frequent pulmonary pediatric diseases.

Results and conclusion LUS has been proven to be a very important diagnostic tool in almost all lung disease in pediatric disease described but obviously still need continuous research to explore its potential.

\section{P557 REVIEW OF INCIDENCE AND INDICATION OF MICTURATING CYSTOURETHROGRAM (MCUG) IN PAEDIATRIC PATIENTS IN CORK UNIVERSITY HOSPITAL (CUH)}

${ }^{1}$ Carol Stephens*, ${ }^{2}$ Sean Flanagan. 'Paediatric Department, Cork University Hospital, Cork, Ireland; ${ }^{2}$ Radiology Department, Cork University Hospital, Cork, Ireland

\subsection{6/archdischild-2019-epa.891}

Micturating Cystourethrogram (MCUG) is commonly uses to investigate Vesocoureteroreflux (VUR). While the test lends itself to a high sensitivity and specificity rate in identification of VUR it doesn't come without risks. The biggest challenge is practicality and tolerability. MCUGs are not pleasant and the window of opportunity to perform them without sedation is narrow. Furthermore, MCUG is an invasive test predisposing the patient to iatrogenic infection. Keeping the above in mind, it is essential that MCUGs are only performed in those who need them and will influence their future management and treatment

\section{Aims}

1. Identify all children in who had a MCUG at less than two years of age

2. Identify the indication for MCUG

3. Investigate if reason for MCUG met guidelines.
Methods A retrospective review of MCUGs performed in CUH in all children less than two years of ages between 01/ 01/15-31/12/17.Patients from Cork University Maternity Hospital were included.Patients were identified via IMPAX Radiology system. Nice Guidelines were used as the standard.

Results

Ninety nine children were identified

\begin{tabular}{lc}
\hline Appropriate Indications & 95 \\
\hline Inappropriate & 4 \\
\hline Indications & \\
\hline
\end{tabular}

\begin{tabular}{ll}
\hline INDICATION & NUMBER \\
\hline Atypical UTI & 20 \\
Recurrent UTI & 1 \\
Hydronephrosis & 68 \\
Scarring & 1 \\
Other & 5 \\
\hline
\end{tabular}

- Two children met more than one indication; atypical UTIs and hydronephrosis

- One child had more than three indications; hydronephrosis, scarring and 'other'

The main reasonfor not meeting criteria was for recurrent UTIs outside the $<6$ month of age period.

Discussion MCUG is recommended in children who have had a renal ultrasound revealing hydronephrosis, scarring or other findings that may suggest high grade reflux or in children less than six months with atypical and or recurrent UTIs. There is considerable controversy regarding the optimal management of VUR varying between prophylactic antibiotics versus surgery. Early identification optimizes kidney preservation. By defining risk, early stratification allows earlier identification of high risk children .Incidence of VUR has been difficult to ascertain but it has been estimated that $0.8-1.4 \%$ of normal children will have reflux. This incidence soars to $30-50 \%$ in children with UTIs depending on age and a higher incidence in males despite a higher incidence of UTIs in females.

In our hospital, we adhere to current best medical practice and don't perform MCUGs when not indicated. This is important given the significant risks associated with MCUGs.

Conclusion The majority of MCUGs were performed in line with current guidelines. The most common indication was hydronephrosis. There is good knowledge among paediatric and radiology teams of the best practice.

\section{P558 A RETROSPECTIVE STUDY OF MANAGEMENT OF HENOCH SCHONLEIN (HSP) NEPHRITIS IN CHILDREN IN IRELAND A}

Carol Stephens*, Tracy Conlon, Mary Waldron, Maria Stack. OLCHC, Dublin, Ireland

10.1136/archdischild-2019-epa.892

HSP is a prevalent disease with an incidence of 6-24 per 100,000.(1) It can lead to significant chronic disease with 